\title{
Impact of COVID-19 Pandemic on Training: Global Perceptions of Gastroenterology and Hepatology Fellows in the USA
}

\author{
Kofi Clarke ${ }^{1}$ (1) - Mohammad Bilal ${ }^{2}$. Sergio A. Sánchez-Luna ${ }^{3}$. Shannon Dalessio ${ }^{1}$. Jennifer L. Maranki ${ }^{1}$. \\ Shazia Mehmood Siddique ${ }^{4}$
}

Received: 9 July 2020 / Accepted: 5 October 2020 / Published online: 19 October 2020

(c) Springer Science+Business Media, LLC, part of Springer Nature 2020

\begin{abstract}
Background The COVID-19 pandemic has impacted numerous facets of healthcare workers' lives. There have also been significant changes in Gastroenterology (GI) fellowship training as a result of the challenges presented by the pandemic.

Aims We conducted a national survey of Gastroenterology fellows to evaluate fellows' perceptions, changes in clinical duties, and education during the pandemic.

Methods A survey was sent to Gastroenterology (GI) fellows in the USA. Information regarding redeployment, fellow restriction in endoscopy, outpatient clinics and inpatient consults, impact on educational activities, and available wellness resources was obtained. Fellows' level of agreement with adjustments to clinical duties was also assessed.

Results One hundred and seventy-seven Gastroenterology fellows responded, and 29.4\% were redeployed to non-GI services during the pandemic. COVID-19 impacted all aspects of GI fellowship training in the USA (endoscopy, outpatient clinics, inpatient consults, educational activities). Fellows' level of agreement in changes to various aspects of fellowship varied. $72.5 \%$ of respondents reported that their programs provided them with increased wellness resources to cope with the additional stress during the pandemic. For respondents with children, 17.6\% reported no support with childcare.

Conclusions Our results show that the COVID-19 pandemic has impacted GI fellowship training in the USA in multiple domains, including gastrointestinal endoscopy, inpatient consults, outpatient clinics, and educational conferences. Our study highlights the importance of considering and incorporating fellows' viewpoints, as changes are made in response to the ongoing pandemic.
\end{abstract}

Keywords Training $\cdot$ Fellowship $\cdot$ Education $\cdot$ COVID-19 pandemic $\cdot$ Gastroenterology fellowship

Electronic supplementary material The online version of this article (https://doi.org/10.1007/s10620-020-06655-y) contains supplementary material, which is available to authorized users.

Kofi Clarke

kclarke@pennstatehealth.psu.edu

1 Division of Gastroenterology and Hepatology, Pennsylvania State University College of Medicine, Hershey, PA, USA

2 Division of Gastroenterology and Hepatology, Beth Israel Deaconess Medical Center, Harvard Medical School, Boston, MA, USA

3 Division of Gastroenterology, Hepatology and Nutrition, Allegheny Health Network, Pittsburgh, PA, USA

4 Division of Gastroenterology, University of Pennsylvania Perelman School of Medicine, Philadelphia, PA, USA

\section{Introduction}

The COVID-19 pandemic has impacted numerous facets of healthcare workers' lives in the USA, as the country with the highest number of reported cases worldwide [1]. While much of the current dialogue is focused on clinical care, an important area of consideration is the impact of the pandemic on training. For Gastroenterology (GI) fellowship programs, adjustments to endoscopy services, inpatient consults, outpatient clinics, and educational conferences have been made in an effort to limit exposure and promote safety [2-4]. However, data on the impact of the COVID-19 pandemic on GI fellowship training in the USA are lacking. We conducted a national survey of GI fellows to evaluate fellows' perceptions, changes in clinical duties, and education during the pandemic. 


\section{Methods}

A 30-item survey was distributed among GI fellows enrolled in Accreditation Council for Graduate Medical Educationaccredited programs in the USA via GI fellowship directors or coordinators (supplement). Data were collected and managed using the REDCap software (supplement) [5]. The institutional review board at the Penn State Hershey Medical Center approved the study. Data on respondent and program demographics and characteristics were collected. Fellows' level of agreement with adjustments to clinical duties was assessed using a 5-point Likert scale from strongly disagree to strongly agree. Agreement was defined as a response of agree or strongly agree. Descriptive statistics were calculated using frequencies and percentages. Multivariable regression analysis was performed to identify predictors of respondents' agreement for involvement in COVID-19 patient care. Variables included were the presence of COVID surge in the hospital, age, sex, level of training, and program size.

\section{Results}

There were 177 respondents, mean age was 32.3 years, and $43.4 \%$ were females. There were 53 first-year, 69 second-year, 50 third-year, and five fourth-year GI fellow respondents; the majority $(83.5 \%)$ were from university programs (Table 1).

\section{Redeployment to Non-GI Services}

Preparations for potential redeployment to non-GI services such as intensive care units (ICU) and medical wards were made for $73 \%$ of respondents, and $29.4 \%$ reported getting redeployed. The majority (91\%) of respondents agreed with redeployment plans, but of those, $64.8 \%(105 / 162)$ believed redeployment should only occur if ICU/medicine/ ER physicians were not available.

\section{Involvement in Care of COVID-19 Patients}

$68.5 \%$ of respondents believed they should be involved in GI care of COVID-19 patients. On a multivariate regression analysis, the only independent positive predictor for fellow agreement to participate in care of COVID-19 patients was a surge of COVID-19 cases at their institution [OR: $2.1,(95 \%$ CI 1.1-4.3), $p=0.03$ ]. There were no statistically significant differences based on age, sex, level of training, and program size.
Table 1 Patient and program-level characteristics

\begin{tabular}{|c|c|}
\hline & Total $(n=177)$ \\
\hline Mean age (years) & 32.2 \\
\hline \multirow[t]{2}{*}{ Sex } & Male $=99(56.6 \%)$ \\
\hline & Female $=76(43.4 \%)$ \\
\hline \multirow[t]{3}{*}{ Relationship status } & Single $=45(25.3 \%)$ \\
\hline & $\begin{array}{l}\text { Married/domestic relation- } \\
\text { ship }=132(74.2 \%)\end{array}$ \\
\hline & Divorced $=1(0.6 \%)$ \\
\hline \multirow[t]{2}{*}{ Children ( $>18$ years of age) } & Yes $=53(29.8 \%)$ \\
\hline & $\mathrm{No}=125(70.2 \%)$ \\
\hline \multirow[t]{4}{*}{ Fellowship year } & 1st: $53(29.9 \%)$ \\
\hline & 2nd: $69(39.0 \%)$ \\
\hline & 3rd: $50(28.2 \%)$ \\
\hline & 4th: $5(2.8 \%)$ \\
\hline \multirow[t]{3}{*}{ Type of fellowship program } & University: 147 (83.5\%) \\
\hline & University-affiliated: $20(11.4 \%)$ \\
\hline & Community hospital: 9 (5.1\%) \\
\hline \multirow{4}{*}{$\begin{array}{l}\text { Number of general Gastroenterol- } \\
\text { ogy fellows in program }\end{array}$} & $1-5: 23(12.9 \%)$ \\
\hline & $6-10: 61(34.3$ \\
\hline & $11-15: 86(48.3 \%)$ \\
\hline & $>15: 8(4.5 \%)$ \\
\hline
\end{tabular}

\section{Impact on Gastrointestinal Endoscopy}

$53.9 \%$ of respondents reported partial restriction in endoscopic procedures (e.g., fellows could participate while on call, but not for routine cases), while $18.5 \%$ reported complete restriction. Only $35.7 \%$ of respondents agreed with this restriction, and $64.3 \%$ of respondents believe that this will impact their endoscopic skills at the end of their training (Fig. 1).

\section{Inpatient Consults}

Regarding inpatient consults, about half $(51.7 \%)$ of the respondents reported no restrictions, while $46.6 \%$ had partial restrictions (not allowed to see COVID-19 patients). Among respondents with complete or partial restriction, $63.8 \%$ agreed with this decision.

\section{Outpatient Clinics}

GI fellows' involvement in telemedicine clinics was reported by $74 \%$ of respondents, and of those, $70.8 \%$ of respondents agreed with this decision. 


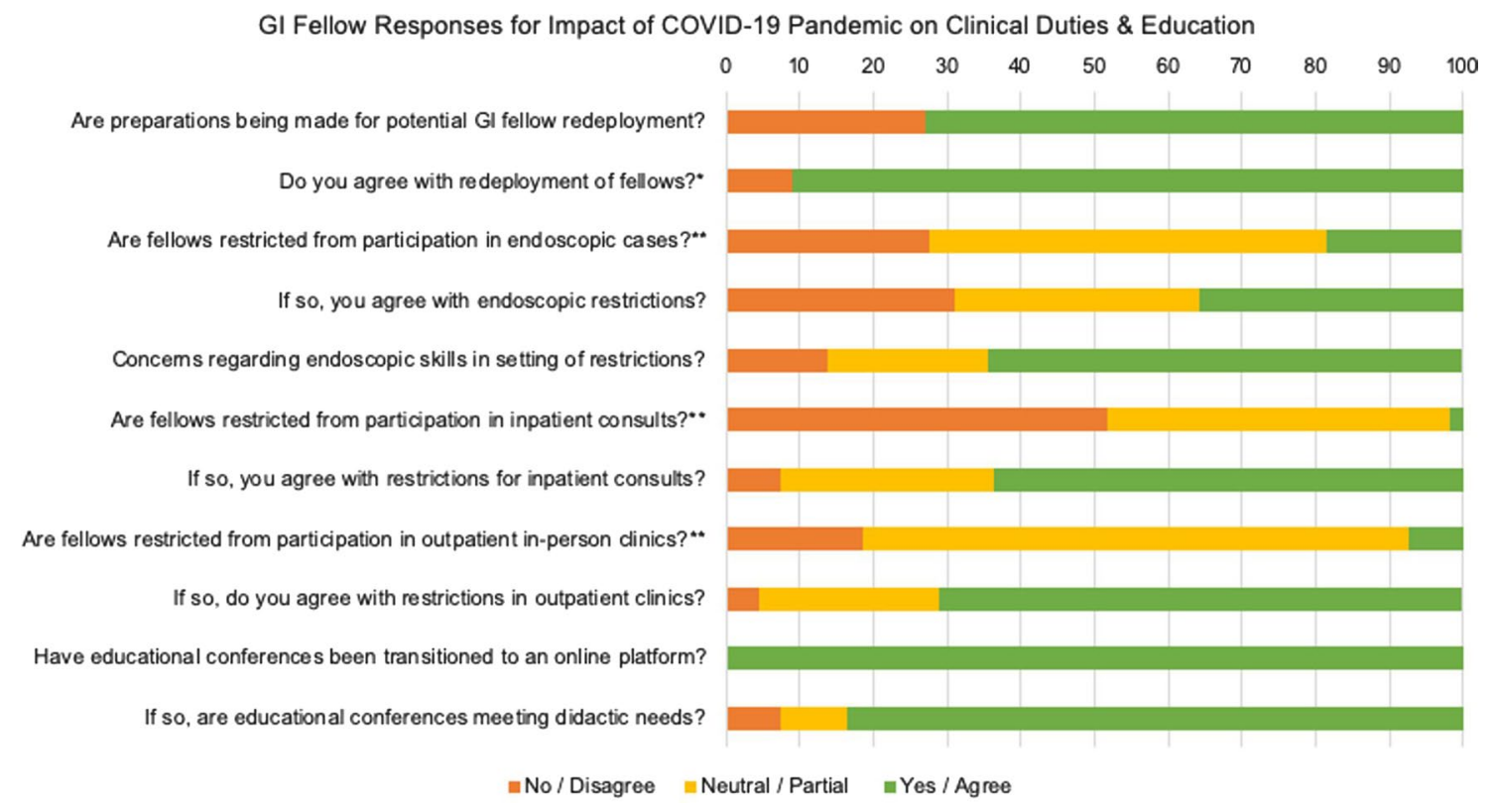

Fig. 1 GI fellow responses for impact of COVID-19 pandemic on clinical duties and education. *Of participants who agreed with redeployment $(n=162), 64.8 \%$ believed redeployment should only occur if ICU/EM/medicine attendings were not available to cover those services. **Partial restriction is defined as follows: For endoscopy, par-

\section{Educational Conferences and Didactics}

All respondents (100\%) reported that their programs had moved to online/virtual didactics, and 53.7\% thought this met their educational needs in the same manner as before, while $34.3 \%$ thought this system was improved.

\section{Wellness Resources Including Childcare}

$72.5 \%$ of respondents reported that their programs provided them with increased wellness resources to cope with the additional stress during the pandemic. For respondents with children, $17.6 \%$ reported either no support or some support with childcare but harder than usual (64.7\%). Most (86\%) of respondents reported that their fellowship program is not delaying graduation for senior fellows as a result of this pandemic.

\section{Discussion}

Our study is the largest survey of US GI fellows during the pandemic and provides important insight on the impact of the COVID-19 pandemic on various aspects of GI fellowship training, as well as information on fellows' perception of resulting changes. In addition, we evaluated the availability of wellness support and resources for trainees. ticipation in endoscopy is allowed while on call, but routine participation is restricted. For inpatient consults, participation in inpatient consults is restricted while caring for COVID-19 patients only. For outpatient clinics, participation for in-person clinic visits is restricted, but participation in telemedicine is allowed

Our results confirm the COVID-19 pandemic has impacted GI fellowship training in the USA in multiple domains, including endoscopy, inpatient consults, outpatient clinics, and educational conferences. Moreover, approximately $30 \%$ of the GI fellow respondents were redeployed to non-GI services during the pandemic. While recent efforts have highlighted this impact [6], and proposed strategies to cope with these challenges [2], there was previously no knowledge as to how US GI fellows are perceiving this impact on their training. Our analysis demonstrates that most respondents agreed with redeployment if ICU and/or medicine attendings were unavailable to cover these nonGI services. This sentiment was also supported by recent guidance from the American Society for Gastrointestinal Endoscopy [7].

While the impact has been greatest on endoscopic procedures, with $72.4 \%$ of respondents reporting some type of restriction on endoscopic participation, only about a third of respondents believe that such a restriction is warranted, and the majority are concerned about the impact on endoscopic competency. The impact on trainee involvement in gastrointestinal endoscopy is not surprising. During the peak of the pandemic, there was an overall reduction in gastrointestinal endoscopy volume $[6,8]$. This was because national gastroenterological organizations as well as experts suggested that all elective and non-urgent endoscopic procedures should be deferred to preserve personal protective equipment (PPE) 
and mitigate the spread of infection [9, 10]. An international survey of endoscopy trainees also found that $93 \%$ of endoscopy trainee respondents reported an overall reduction in endoscopy volume [6]. The survey also evaluated barriers to trainee involvement in endoscopy and found changes in institutional policy as the most common reason for decrease in trainee involvement, followed by lack of cases, shortage of PPE, and redeployment to other clinical areas [6]. Other reports have also shown that there was significant reduction in gastrointestinal endoscopy volume during the pandemic $[8,11]$. We also found that majority of GI fellows are concerned regarding achieving competence in endoscopic skills due to these restrictions. Therefore, consideration to resume fellow participation in endoscopy as local prevalence decreases should take into account fellows' desires and concerns for achieving adequate endoscopic competency.

Our results also showed that majority of fellows are participating in some form of telehealth services provided to patients. These findings are similar to other recent reports which have also shown that majority of gastroenterologists in the USA have adopted telehealth in their clinical practice [12]. In contrast to restrictions in endoscopy, the restrictions on involvement in inpatient and outpatient clinical duties were supported by the majority of respondents.

Our study has several limitations. Only 177 of the GI fellows responded; however, this number is comparable to recently published studies focused on GI fellows [13-15]. Our survey does not provide exact numbers of procedures or clinic days missed as a result of the pandemic, and the results demonstrate one-time cross-sectional view of GI fellows' perception of changes to their fellowship during the current pandemic.

While the COVID-19 pandemic has led to an inevitable impact on GI fellowship training, our study highlights the importance of considering and incorporating fellows' viewpoints, as changes are made in response to the ongoing pandemic. Furthermore, all programs should make efforts to provide additional wellness resources, particularly relating to childcare, during the pandemic.

Acknowledgment The authors acknowledge the assistance of Venkata Gorrepati, MD, for assistance with data analysis.

Authors' contribution KC contributed to conceptualization, data curation, formal analysis, investigation, methodology, and writing-original draft. MB helped with conceptualization, data curation, investigation, methodology, and writing - original draft. SAS-L contributed to conceptualization and writing-original draft. SD helped with data curation and formal analysis. JLM contributed to conceptualization, methodology, and writing-review and editing. SMS helped with conceptualization, supervision, investigation, methodology, and writing-review and editing.

Funding The authors received no financial support or grants for the research, authorship, and publication of this article.

\section{Compliance with Ethical Standards}

Conflicts of interest Kofi Clarke has the following disclosures: Pfizer-Research Grant Reviewer/Consultant/Speaker's Bureau, Janssen-Speaker's Bureau, Takeda-Speaker's Bureau, and ABBVieSpeaker's Bureau. The remaining authors have no conflicts of interest.

Research involving human participants and/or animals The study was approved by the institutional review board (IRB) at Penn State Hershey Medical Center, PA. All reporting was in compliance with ethical standards.

Informed consent Informed consent was obtained.

\section{References}

1. Johns Hopkins Corona Virus Resource Center. https://coronaviru s.jhu.edu/map.html. Accessed May 14, 2020.

2. Keswani RN, Sethi A, Repici A, et al. How to maximize trainee education during the COVID-19 pandemic: perspectives from around the world. Gastroenterology. 2020;159:26-29.

3. Mallepally N, Bilal M, Hernandez-Barco YG, et al. The new virtual reality: how COVID-19 will affect the gastroenterology and hepatology fellowship match. Dig Dis Sci. 2020;65:2164-2168. https://doi.org/10.1007/s10620-020-06432-x.

4. Shah R, Satyavada S, Ismail M, et al. COVID-19 pandemic through the lens of a gastroenterology fellow: looking for the silver lining. Gastrointest Endosc. 2020;92:394-398.

5. Harris PA, Taylor R, Thielke R, et al. Research electronic data capture (REDCap) - a metadata-driven methodology and workflow process for providing translational research informatics support. J Biomed Inform. 2009;42:377-381.

6. Pawlak KM, Kral J, Khan R, et al. Impact of COVID-19 on endoscopy trainees: an international survey. Gastrointest Endosc. 2020;92:925-935.

7. Bowman D. American Society for Gastrointestinal Endoscopy: guidance for trainees during the COVID-19 pandemic. Gastrointest Endosc. 2020;92:748-753.

8. Parasa S, Reddy N, Faigel DO, et al. Global impact of the COVID-19 pandemic on endoscopy: an international survey of 252 centers from 55 countries. Gastroenterology. 2020; https:// doi.org/10.1053/j.gastro.2020.06.009.

9. Sultan S, Lim JK, Altayar O, et al. AGA Rapid Recommendations for Gastrointestinal Procedures During the COVID-19 Pandemic. Gastroenterology. 2020;159:739-758.e4.

10. Sawhney MS, Bilal M, Pohl H, et al. Triaging advanced GI endoscopy procedures during the COVID-19 pandemic: consensus recommendations using the Delphi method. Gastrointest Endosc. 2020;92:535-542.

11. Mahadev S, Aroniadis OS, Barraza L, et al. Impact of the COVID19 pandemic on endoscopy practice: results of a cross-sectional survey from the New York metropolitan area. Gastrointest Endosc. 2020;92:788-789.

12. Keihanian T, Sharma P, Goyal J, et al. TeleHealth utilization in Gastroenterology (GI) clinics amid Coronavirus-19 (COVID-19) pandemic: impact on clinical practice AND GI training. Gastroenterology. 2020; https://doi.org/10.1053/j.gastro.2020.06.040.

13. Kumar NL, Perencevich ML, Trier JS. Perceptions of the inpatient training experience: a nationwide survey of gastroenterology program directors and fellows. Dig Dis Sci. 2017;62:2631-2647. https://doi.org/10.1007/s10620-017-4711-y. 
14. Ordway SM, Singla MB, Young PE, et al. Factors influencing decisions about a career in hepatology: a survey of gastroenterology fellows. Hepatol Commun. 2017;1:347-353.

15. Villa E, Attar B, Trick W, et al. Endoscopy-related musculoskeletal injuries in gastroenterology fellows. Endosc Int Open. 2019;7:E808-e812.
Publisher's Note Springer Nature remains neutral with regard to jurisdictional claims in published maps and institutional affiliations. 